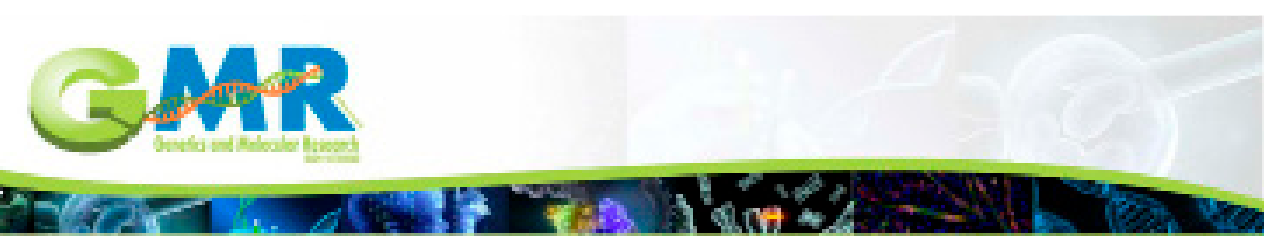

\title{
Genetic diversity analysis of fruit characteristics of hawthorn germplasm
}

\author{
K. Su*, Y.S. Guo*, G. Wang, Y.H. Zhao and W.X. Dong \\ College of Horticulture, Shenyang Agriculture University, Shenyang \\ *These authors contributed equally to this study. \\ Corresponding authors: Y.H. Zhao / W.X. Dong \\ E-mail: zhaoyuhui76@126.com / wxdong63@126.com
}

Genet. Mol. Res. 14 (4): 16012-16017 (2015)

Received June 30, 2015

Accepted September 25, 2015

Published December 7, 2015

DOI http://dx.doi.org/10.4238/2015.December.7.14

\begin{abstract}
One hundred and six accessions of hawthorn intraspecific resources, from the National Germplasm Repository at Shenyang, were subjected to genetic diversity and principal component analysis based on evaluation data of 15 fruit traits. Results showed that the genetic diversity of hawthorn fruit traits varied. Among the 15 traits, the fruit shape variable coefficient had the most obvious evaluation, followed by fruit surface state, dot color, taste, weight of single fruit, sepal posture, peduncle form, and metula traits. These are the primary traits by which hawthorn could be classified in the future. The principal component demonstrated that these traits are the most influential factors of hawthorn fruit characteristics.
\end{abstract}

Key words: Hawthorn; Genetic diversity; Principal component 


\section{INTRODUCTION}

Hawthorn (Crataegus spp) is native to China, and over the past 3000 years, by both natural and artificial (human) selection, it has developed to form abundant germplasm. In 1982, the unique professional hawthorn germplasm resource-keeping garden was constructed in Shenyang, China. Four hundred accessions, contained in 11 Crataegus species, have been collected and saved in this repository; most resources belong to the intraspecific cultivar of hawthorn. As the scale of the collecting resource increases, research and appraisal of basic traits of hawthorn are possible. Germplasm resource evaluation has been carried out on the majority of fruit tree species in China, especially with respect to the study of fruit traits. Data has been widely reported for most species, including macadamia nut (Wang et al., 2011); loquat (Lin et al., 2009; Zhang et al., 2009); jack fruit (Wang et al., 2009); mango (Zhu et al., 2010); longan (Huang et al., 2010); Jujube (Yang et al., 2006); strawberry (Yang et al., 2007); apple (Wang et al., 2007); cherry (Qi et al., 2008); and peach (Wang et al., 2005). The evaluation of hawthorn germplasm resource, however, has not been reported. In the current study, genetic diversity and principal component was analyzed to evaluate fruit traits for 106 accessions of Chinese hawthorn. The study provides practical guidance and theoretical basis for the future collection, development, and variety breeding of hawthorn.

\section{MATERIAL AND METHODS}

\section{Plants}

Accessions of hawthorn intraspecific resources $(N=106)$, maintained in the Shenyang National Hawthorn Germplasm Nursery were used (Table 1). The picking date was October of 2012. For each specimen, 15 fruit traits were evaluated.

Table 1. Hawthorn (Crataegus pinnatifida Bge.) cultivars included for analysis.

\begin{tabular}{llllll}
\hline Resource name & Resource name & Resource name & Resource name & Resource name & Resource name \\
\hline Anshanzirou & Fushantieqiu & Liaohong & Pingyitianhongzi & Waibahong & Yiduxiaohuang \\
Baili & Guajiayu No. 1 & Liaoyangzirou & Pingyiyinhongzi & Wanqiushanlihong & Yinyeling No. 2 \\
Bairangmian & Haitangshanzha & Linxianshangkou & Qingyuanmopan & Wanbaodidajinxing & Yinyeling No. 9 \\
Baihuayudahong & Hanfeng & Linfen No. 1 & Qiufeng & Wanbaodishisheng & Zaohong \\
Baihuayushanzha & Hezedashanzha & Lufu No. 1 & Qiuhong & Xifen No. 1 & Zimuhong \\
Beijingshanzha & Huabeixiaoshanzha & Luojiagoushanzha & Qiujinxing & Xifen No. 4 & Zizhenzhu \\
Benxi No. 7 & Hengrenxiangyang & Maershanlihong & Qiuli & Xifen No. 5 & Fengshuishanzha \\
Chaojixing & Huangbaoyu No. 1 & Magangzaohong & Shandongdajinxing & Xifenshisheng & Laiwuheihong \\
Dahuang & Huangguo & Majiadadui & Shanxitiansheng & Xifenghong & Pingyishanzha \\
Dahuo & Huixiandahongkongqi & Majiafenrou & Shangzhuanbaizha & Xihong & Tongtaibaiyesheng \\
Damianqiu & Jiguanshanshanzha & Mengyinbanyexiaojinxing & Shenglizirou & Xiajinxing & Yiduchangkou \\
Datangqiu & Jilinyehe & Mengyindajinxing & Shisheng No. 1 & Xianpingzhenmu No. 1 Feixiandamianqiu \\
Dawangmiao & Jianzirou & Mengyinxiaojinxing & Shuanghong & Xiaomianqiu & Kaiyuanruanzi \\
Dawang & Jianchangshanzha & Muhuli & Songshancunshisheng & Xinghong No. 3 & Pingyifuhongzi \\
Donglingqingkou & Jinxiandahongshanzha & Niejiayu No. 2 & Taianshiliu & Xinglongshisheng & Tongliaohong \\
Donglingshanzha & Jinxiantianshanzha & Niuxintai No. 1 & Tangchi No. 2 & Xingshiliu & Yepu No. 1 \\
Duanzhihanluhong & Jinxianxiaoye & Piposhi & Tianshui & Yanrangqing \\
Fakushishengshanlihong & Jiuzhaishanzha & Pingyidahongzi & Tielingshanzha & Yangjiabaodahong \\
\hline
\end{tabular}

\section{Data collection, trimming, and analysis}

Based on the Hawthorn Germplasm Resource Description and Data Standard, basic trait and identification data were collected for each accession, including 14 fruit classification traits 
(peduncle form and trait; sepal form and posture; fruit form, surface color and surface state; fruit dot quantity, color, and size; pulp color and texture, and fruit flavor and fragrance) and a single numeric trait (fruit weight). The classification code used is shown in Table 2. All data were analyzed using SPSS statistical software.

\begin{tabular}{|c|c|c|c|c|c|c|c|c|c|}
\hline \multirow[t]{2}{*}{ Characteristic } & \multirow{2}{*}{$\begin{array}{l}\text { No. of } \\
\text { cultivars }\end{array}$} & \multicolumn{8}{|c|}{ Grade code } \\
\hline & & 1 & 2 & 3 & 4 & 5 & 6 & 7 & 8 \\
\hline Peduncle form & 106 & Wide shallow & Flat & Upheaval & & & & & \\
\hline Metula trait & 106 & Expanded & Tumor in one side & & & & & & \\
\hline Sepal shape & 106 & Triangular & Lanceolate & Ligulate & & & & & \\
\hline Sepal posture & 93 & $\begin{array}{l}\text { Open, sepal } \\
\text { apex upright }\end{array}$ & $\begin{array}{l}\text { Half open, sepal } \\
\text { apex upright }\end{array}$ & $\begin{array}{l}\text { Half open, sepal } \\
\text { apex warped }\end{array}$ & $\begin{array}{l}\text { Opening, sepal } \\
\text { apex flat }\end{array}$ & $\begin{array}{l}\text { Opening, sepal } \\
\text { apex warped }\end{array}$ & Sepal folding & $\begin{array}{l}\text { Folding, sepal } \\
\text { apex warped }\end{array}$ & \\
\hline Fruit shape & 100 & Sub-rounded & Oblate & Ovate & Obovate & Oblong & Wide ovate & Squarish & Irregular \\
\hline Surface color & 99 & Yellow & Orange red & Red & & & & & \\
\hline Surface state & 99 & Rough & Smooth matte & Smooth glossy & & & & & \\
\hline Fruit dot quantity & 94 & None & Few & Medium & Many & & & & \\
\hline Fruit dot color & 95 & Ashen & Golden & Tawny & Brown & & & & \\
\hline Fruit dot size & 87 & Small & Medium & Large & & & & & \\
\hline Sarcocarp color & 106 & Green & White & Yellow & Pink & Red & Purple & & \\
\hline Fruit texture & 106 & Hard & Compact & Mellow-soft & Soft & & & & \\
\hline Fruit flavor & 106 & Sweet & Sour and sweet & Sweet and sour & Sour & Sourest & Thin & Bitter & \\
\hline Fruit fragrance & 106 & None & Thin & Thick & & & & & \\
\hline
\end{tabular}

\section{RESULTS}

\section{Genetic diversity analysis of hawthorn germplasm fruit characteristics}

Results are shown in Tables 3 and 4. The variable coefficient of the fruit form (100 accessions) had the highest value (54.88\%); fruit form was diverse and included suborbicular, flat circular, ovate, obovate, oblong, oval, wide ovate and square forms. The variable coefficient of the fruit surface state (99 accessions) was 42.87\%; this trait included rough, smooth glossy, and smooth matte classifications. The variable coefficient of fruit dot color was $42.29 \%$, including ashen, golden, tawny, and brown classifications (95 accessions). The variable coefficient of the peduncle form was $37.74 \%$; wide-shallow, flat and upheaval forms were described in the 106 accessions. The variable coefficient of sepal posture was $37.42 \%$ and included open-upright, half open-upright, half open-warped, open-flat, open-warped, folding, and folding-warped classifications. The variable coefficient of metula trait was $35.51 \%$, including the classifications expanding, and a tumor in one side. The variable coefficient of fruit dot size was $31.75 \%$, including small, medium, and large. The variable coefficient of sepal trait was $23.54 \%$, including trilateral, lanceolate, and ligulate forms. The variable coefficient of fruit dot number was $20.56 \%$, including few, medium, and many. The variable coefficient of fruit surface color was $17.22 \%$, including yellow, red, and orange red. The variable coefficient of fruit fragrance was $41.39 \%$, including thick, thin, and without smell. The variable coefficient of pulp color was $34.18 \%$, including green, white, yellow, pink, and red. The variable coefficient of fruit fragrance was $26.51 \%$ including thin, thick, and without fragrance. The variable coefficient of fruit texture was $23.87 \%$, including hard, compact, mellow soft, and soft. The variable coefficient of single fruit weight was 36.15\%; the amplitude of variation was $1.5-16.5 \mathrm{~g}$. Significant variable coefficients were those of fruit shape, fruit surface, fruit dot color, fruit flavor, single fruit weight, sepal posture, peduncle form and the trait of metula. Based on these results, we suggest that these be the major traits for hawthorn categorization in the future. 
Table 3. Genetic diversity analysis of hawthorn (Crataegus pinnatifida Bge.) fruit traits.

\begin{tabular}{|c|c|c|c|c|c|c|}
\hline Character & No. of cultivars & Min & Max & Mean & SD & $\mathrm{CV}$ \\
\hline Peduncle form & 106 & 1.00 & 3.00 & 1.4057 & 0.53056 & 37.74 \\
\hline Metula trait & 106 & 1.00 & 2.00 & 1.3208 & 0.46898 & 35.51 \\
\hline Sepal shape & 106 & 1.00 & 3.00 & 2.3585 & 0.55530 & 23.54 \\
\hline Sepal posture & 93 & 1.00 & 7.00 & 3.9785 & 1.48893 & 37.42 \\
\hline Fruit shape & 100 & 1.00 & 8.00 & 4.8200 & 2.64529 & 54.88 \\
\hline Surface color & 99 & 1.00 & 3.00 & 2.8384 & 0.48879 & 17.22 \\
\hline Surface state & 99 & 1.00 & 3.00 & 1.4141 & 0.60626 & 42.87 \\
\hline Fruit dot quantity & 94 & 2.00 & 4.00 & 3.2447 & 0.66698 & 20.56 \\
\hline Fruit dot color & 95 & 1.00 & 4.00 & 2.6105 & 1.1041 & 42.29 \\
\hline Fruit dot size & 87 & 1.00 & 3.00 & 1.9425 & 0.61675 & 31.75 \\
\hline Sarcocarp color & 106 & 1.00 & 5.00 & 2.8491 & 0.97387 & 34.18 \\
\hline Fruit texture & 106 & 1.00 & 4.00 & 2.8585 & 0.68227 & 23.87 \\
\hline Fruit flavor & 106 & 1.00 & 7.00 & 3.1226 & 1.29249 & 41.39 \\
\hline Fruit fragrance & 106 & 1.00 & 3.00 & 2.2264 & 0.59013 & 26.51 \\
\hline e & 106 & 1.50 & 16.25 & 7.6963 & 2.78245 & 36.15 \\
\hline
\end{tabular}

\begin{tabular}{|c|c|c|c|c|c|c|c|c|c|}
\hline \multirow[t]{2}{*}{ Character } & \multirow[t]{2}{*}{ No. of cultivars } & \multicolumn{8}{|c|}{ Frequency } \\
\hline & & 1 & 2 & 3 & 4 & 5 & 6 & 7 & 8 \\
\hline Peduncle form & & 106.0 & 61.32 & 36.79 & 1.88 & & & & \\
\hline Metula trait & 106 & 67.92 & 32.07 & & & & & & \\
\hline Sepal shape & 106 & 3.77 & 56.60 & 39.62 & & & & & \\
\hline Sepal posture & 93 & 7.52 & 6.45 & 17.20 & 38.70 & 18.27 & 3.22 & 8.60 & \\
\hline Fruit shape & 100 & 5.0 & 37.0 & 1.0 & 2.0 & 1.0 & 8.0 & 29.0 & 17 \\
\hline Surface color & 99 & 5.05 & 6.06 & 88.88 & & & & & \\
\hline Surface state & 99 & 64.64 & 29.29 & 6.06 & & & & & \\
\hline Fruit dot quantity & 94 & 0.0 & 12.76 & 50.0 & 37.23 & & & & \\
\hline Fruit dot color & 95 & 17.89 & 33.68 & 17.89 & 30.52 & & & & \\
\hline Fruit dot size & 87 & 21.83 & 62.06 & 16.09 & & & & & \\
\hline Sarcocarp color & 106 & 16.98 & 3.77 & 57.54 & 20.75 & 0.94 & & & \\
\hline Fruit texture & 106 & 2.83 & 22.64 & 60.37 & 14.45 & & & & \\
\hline Fruit flavor & 106 & 6.60 & 25.47 & 37.73 & 17.92 & 6.60 & 2.83 & & \\
\hline Fruit fragrance & 106 & 8.49 & 60.37 & 31.31 & & & & & \\
\hline
\end{tabular}

\section{Analysis of frequency distribution of hawthorn traits}

Genetic diversity of fruit traits in 10 hawthorn germplasm resource (Table 4) demonstrated that the peduncle form for most fruits was wide-shallow $(61.32 \%)$, followed by open and flat; upheaval was the least common form. For the majority of accessions, the metula trait was expanded. The most common sepal state was triangular (56.6\% of cases), followed lanceolate and then ligulate. The most common sepal posture was open and flat, followed by half open warped and open warped; open straight, half open straight and folded were less common, and folded warped was the least common. The majority of accessions had oblate fruit, and the most common fruit color was red (in $88.88 \%$ of cases). The most common fruit surface trait rough. All fruit examined had dots; the most common classification for the number of dots was medium, the majority of dots were golden and brown, and the majority of dots were medium sized. The most common sarcocarp color was yellow. The most common fruit texture was soft; the majority of fruit had a sour sweet or sweet sour flavor, and a thin fragrance $(60.37 \%)$. 


\section{Principal component analysis}

Principal component analysis demonstrated the effect of each factor in form diversity; the cumulative contributive percentage of the first six principal components was $65.472 \%$, containing the vast majority of messages (Table 5). The contributive percentage of the first principal component was $19.004 \%$, of which, single fruit weight had a significant capacity, being the main factor of fruit economic traits. The second principal component included peduncle depression, metula and fruit dot color; these traits had abundant genetic diversity (Table 3). The third principal component included fruit texture, fruit flavor and sarcocarp color; these may reflect the correlated character of fruit texture in which fruit flavor is a negative factor. The fourth principal component included sepal posture and fruit surface traits; the fifth included fruit and sepal shape, with sepal shape being a negative factor; the sixth included fruit surface color, dot size, and dot numbers, with the latter being a negative factor.

Table 5. Results of analysis of principal components in hawthorn.

\begin{tabular}{|c|c|c|c|c|c|c|c|}
\hline & & \multicolumn{6}{|c|}{ Principal component } \\
\hline & & Prin1 & Prin2 & Prin3 & Prin4 & Prin5 & Prin6 \\
\hline \multicolumn{2}{|l|}{ Eigen value } & 2.661 & 1.687 & 1.433 & 1.316 & 1.058 & 1.011 \\
\hline \multicolumn{2}{|c|}{ Contributive percentage } & 19.004 & 12.052 & 10.234 & 9.397 & 7.559 & 7.224 \\
\hline \multicolumn{2}{|c|}{ Total contributive percentage } & 19.004 & 31.057 & 41.291 & 50.688 & 58.247 & 65.472 \\
\hline \multirow[t]{15}{*}{ Eigenvectors } & Single fruit weight & 0.4653 & -0.1414 & -0.0443 & 0.0463 & -0.0375 & -0.0569 \\
\hline & Sarcocarp color & -0.1976 & -0.3248 & -0.3492 & -0.1843 & 0.3000 & 0.3225 \\
\hline & Fruit texture & -0.1958 & -0.1429 & 0.3877 & -0.3611 & 0.2452 & -0.2951 \\
\hline & Fruit flavor & 0.1371 & 0.2248 & -0.4402 & 0.3824 & -0.1548 & -0.0922 \\
\hline & Fruit fragrance & -0.1910 & -0.3556 & 0.2180 & 0.3157 & -0.1125 & -0.1412 \\
\hline & Peduncle form & -0.2054 & 0.4263 & 0.2934 & 0.2509 & -0.0077 & 0.2088 \\
\hline & Metula trait & -0.1922 & 0.4414 & -0.0885 & 0.1491 & 0.3106 & 0.0294 \\
\hline & Sepal shape & 0.3048 & 0.0609 & 0.2033 & -0.1676 & -0.4346 & 0.0088 \\
\hline & Sepal posture & 0.2713 & -0.1135 & 0.3992 & 0.3954 & -0.0750 & 0.0863 \\
\hline & Fruit shape & 0.2042 & 0.0887 & 0.1598 & 0.2093 & 0.5721 & -0.4333 \\
\hline & Surface color & 0.2485 & 0.0579 & 0.0475 & 0.1287 & 0.3404 & 0.4892 \\
\hline & Surface state & -0.3138 & -0.1789 & 0.1082 & 0.3222 & 0.0125 & -0.0912 \\
\hline & Fruit dot quantity & 0.3012 & -0.2143 & 0.2057 & -0.0426 & 0.2442 & 0.3922 \\
\hline & Fruit dot color & 0.1269 & 0.4459 & 0.2008 & -0.3926 & 0.0019 & -0.0284 \\
\hline & Fruit dot color & 0.3581 & -0.0602 & -0.2664 & -0.0231 & 0.1779 & -0.3775 \\
\hline
\end{tabular}

\section{DISCUSSION}

Analysis of fruit genetic diversity demonstrated that fruit traits reflect abundant genetic variation, with variable coefficients between 17.22 and $54.88 \%$. Fruit shape, which had the highest variable coefficient, is an important indicator to reflect the external character of fruit, as is fruit surface state, which had the next highest variable coefficient. Fruit color had the smallest variable coefficient indicating that the color of the hawthorn is relatively uniform. Based on this result, we recommend increasing the number of yellow and orange cultivars within the hawthorn collection. For the majority of accessions, the sarcocarp was yellow, and followed by pink and green, with white and red cultivars (including Damianqiu, Datangqiu, Hengrenxiangyang, Niejiayu No. 2) being very rare. For the majority of the fruit, the sarcocarp texture was mellow. Hard sources are reportedly very rare (Dong, 2013) and in the current study, hawthorn cultivars that were very hard (including Pingyidahongzi, Shanxitiansheng, and Xifenshisheng) were outstanding resources. The majority of cultivars had a fruit that was sour-sweet or sweet-sour in flavor. Hawthorns which taste 
very sour, sweet, and with thin fragrance were very rare, so the sour-sweet and the sweet-sour are the outstanding resources, and there are 67 shares totally in 106 shares. Thin fruit fragrance was common, and fruits without fragrance were very rare. Based on analysis of frequency distribution of fruit traits in germplasm resources, the quantitative traits sepal posture, fruit shape, fruit dot color, and the number of fruit dots are abundant. There were seven classifications for sepal posture, but the majority of cultivars examined were classified as open and flat; fruit shape had eight classifications, and oblate was the most common; the majority of fruits had a rough surface; hawthorns with a smooth, glossy surface were very rare; and the majority of cultivars had a red fruit color, which species bearing yellow fruits being very rare.

These results demonstrate that hawthorn fruit peduncle, metula, sepal, fruit surface, the fruit dot form, and fruit flavor have genetic diversity. The data presented here provide abundant information on parent materials for hawthorn breeding, and improved scope for the development of hawthorn resources.

\section{Conflicts of interest}

The authors declare no conflict of interest.

\section{ACKNOWLEDGMENTS}

Research supported by the National Natural Science Funds of China (grant \#31101515).

\section{REFERENCES}

Dong WX (2013). Technical specifications for crop germplasm characterization and evaluation of Hawthorn (NY/T 2325-2013). China Agriculture Publishing House.

Huang AP, Chen XP, Hu WS, Jiang F, et al. (2010). Fruit character diversity analysis and numerical classification of longan (Dimocarpus) germplasm resources. J. Fruit Sci. 27: 938-945

Lin QH, Xu QZ, Zheng S, Zhang XJ, et al. (2009). Analysis on the diversity and correlation of fruit bearing in loquat germplasm resources. Fujian Fruits 1: 4-10.

Lv DG and Li ZX (2006). Descriptors and data standard for hawthorn (Crataegus L.). China Agricultural Science and Technology Press.

Qi XJ, Zhao GR, Xu SK, Han LX, et al. (2008). Study on numerical taxonomy of some fruit characteristics of cherry germplasm resources. J. Fruit Sci. 25: 650-654.

Wang K, Liu FZ, Xiao YH, Gong X, et al (2007). Fruit quantitative character evaluation and analysis of apple germplasm. China Fruits 5: 14-17.

Wang LR, Zhu GR and Fang WC (2005). The evaluating criteria of some fruit quantitative characters of peach (Prunus persica I.) genetic resources. Acta Hortic. Sin. 32: 1-5.

Wang W, Yang WH, Zhang MK, Zeng H, et al. (2011). Fruit characteristics of macadamia genetic resources. Chin. J. Trop. Agric. 31: 11-16.

Wang YH, Ye CH, Feng F and Li YZ (2009). Morphological markers and principal components analysis of jackfruit germplasm in Leizhou Peninsula. Chin. J. Trop. Crops 30: 761-768.

Yang L, Zhou JY, Liu P, Zhao ZH, et al. (2006). Variation and probability grading of main quantitative characters of wild jujube germplasm resource. J. Agric. Univ. Hebei 29: 34-37.

Yang L, Yang L, Li L, Sun LY, et al. (2007). Variation and probability grading of main quantitative characters of fruit in strawberry germplasm resource. Southwest China J. Agric. Sci. 20: 1067-1069.

Zhang XJ, Zhang S, Zhang XY, Huang AP, et al. (2009). Research on fruit cluster \& fruit external properties of loquat germplasm resources. Fujian Fruits 2: 63-67.

Zhu M, Gao AP, Deng SS and Chen YY (2010). Evaluation index of main quantitative characters of mango (Mangifera indica) genetic resources. J. Plant Genet. Res. 11: 418-423. 